\title{
Which Buttons And Bars? An Exercise In Community Participation In Decision Support Software Development
}

\author{
S. M. Cuddy., F. M. Marston and T.F.N. Farley. \\ CSIRO Land and Water, GPO Box 1666, ACT 2601 Australia
}

Key words: Ecological river modelling, decision support system, environmental assessment.

Abstract: In developing an environmental DSS it is important to have a good understanding of who will use it and how. The best way to do this is by interacting with a user group during the development process to ensure an appropriate design and to foster interest and ownership of the product. A prototype of the Environmental Flows Decision Support System (EFDSS) has been developed in this manner. Using the Border Rivers Catchment of NSW/QLD as a case study, a community reference group was established and regularly consulted throughout development of the system. Members provided important input and feedback on interface design resulting in numerous modifications and enhancements to the final prototype. Many lessons were learned and one of the spin-offs was the addition of an entire explanation module - EFDSSInfo - which had otherwise only been considered peripheral to the system.

\section{INTRODUCTION}

Throughout Australia's Murray-Darling Basin, State governments are working with communities and agencies to determine water management plans for the rivers of the basin. There is a need to service this process with tools which can assist decision makers in assessing the many flow management options under consideration for regulated river systems. The Environmental Flows Decision Support System (EFDSS) (Young et al, 1999) is such a tool. Using a range of assessment methods, the EFDSS

The original version of this chapter was revised: The copyright line was incorrect. This has been corrected. The Erratum to this chapter is available at DOI: 10.1007/978-0-387-35503-0_29 
provides measures of the likely impact of different flow regimes on the environmental condition of a river system, including its floodplains. Thus, the EFDSS does not predict environmental flows; rather it is a tool for assessing any flow regime which can be described by a set of daily flow files. These flow files must be associated with locations within the river system and its floodplains that are considered ecologically important. Such flow files are normally produced by hydrology models, many of which are currently being developed for the catchments within the Murray-Darling Basin. It was considered that such a tool could make a significant contribution to an informed discussion on balancing environmental and human water use. Because the EFDSS design is pitched directly at community and non-technical users, it was critical to the success of the design and uptake of the software that a wide range of users be involved in its design. Thus an EFDSS community reference group was formed. This was not straight-forward.

Two broad groups of users are targeted in the EFDSS: (i) community members of catchment or river management planning groups with limited technical computing or ecological knowledge, and (ii) staff of various government agencies or private sector companies involved in environmental management who are technically competent computer users, with experience in environmental management and modelling. EFDSS has two modes of operation: the Public User Interface (PUI), and the Technical User Interface (TUI). It is the PUI which is the 'face' of EFDSS and its design was the focus for the reference group.

\section{COMMUNITY PARTICIPATION PROCESS}

As the purpose of the EFDSS was to provide a tool to assist the process of determining appropriate flow management plans for rivers of the MurrayDarling Basin, it was important to understand the decision-making environment. The best way of doing this was to interact with potential users during the development phase to ensure an appropriate design and to foster interest and ownership of the product. This interaction was formalised through the formation of an EFDSS community reference group. The Border Rivers Catchment of NSW/QLD was chosen as a case study. Although a similar process for flow management planning is in place in many of the catchments of the Murray-Darling Basin, the actual implementation is very much a function of the relationships and dynamics amongst the local stakeholders, including government agency staff and water users. While the method of developing a case study is useful in the software design process to provide focus and relevance of software design, it can also inhibit the process by being bound to the peculiarities of the case study. This was the 
case in the Border Rivers where a community reference panel had already been formed to develop an appropriate water allocation management plan, and the dynamics of that body had to be considered when forming the EFDSS reference group. While it was important that the group be representative of those involved in the planning process, the EFDSS development could not interfere with the planning process. Thus the small EFDSS group worked outside the planning process.

\subsection{Formation of the Community Reference Group}

To set up the group, a paper describing the role and requirements of an EFDSS reference group was prepared. This was used as the basis for discussions with relevant government agencies, and the local community reference panel. While the paper suggested that two groups be formed - a group that would serve as a sounding board for the EFDSS, and a smaller group to be more closely involved with the EFDSS design - only the second group was formed from interested members of the community reference panel. The composition of the group was influenced by the coordinator of the panel, to ensure a balance between stakeholders. However, all members were relatively naïve software users. Thirteen people representing a variety of interests (private landholders, catchment management groups, state land and water management agencies and local government) joined the reference group and participated over an 18 month period. The method of interaction with the group was heavily influenced by three factors:

- the purpose was software design, not the Border Rivers case study, ie the case study was for illustrative purposes only;

- the group was physically distant from the software developers;

- it was not possible to distribute the software to members of the group.

Firstly, only a few visits to the catchment by the developers were possible. To ensure maximum outcome from these visits, the software development cycle was modified to deliver a working product at five milestones through the life of the project. Each product built on earlier ones and could thus incorporate feedback received from the group during the visits. Between visits, and for the visits, communication fact sheets were used to keep the group informed and to get feedback, which was usually via telephone, though sometimes written. To notify a wider audience of the progress of the EFDSS, newsletters were produced, and distributed via email to scientists and managers, outside of the Border Rivers. These three forms 
of communications - visits, newsletters and communication fact sheets - are outlined in more detail below.

\subsection{Working with the Reference Group}

The relationship with the group grew over the period of the project and the mode of communication matured. While the original intention was that the group would not be asked to collect data, it became obvious that this was something that the group could and wanted to contribute. So, some of the communication sheets were designed to elicit information on the ecological condition of the Border Rivers. However, it must be reported that the effort made in preparing communications was not matched with feedback from the group. By far the best feedback was elicited from direct interaction with the members during the visits to the catchment. Aspects identified that would benefit from reference group participation through input and feedback included:

- local issues and questions

- software design

- defining who would use EFDSS

- determining how much hydrological knowledge a user need have compared with how much should be provided by EFDSS itself

- determining the questions that EFDSS needed to address

- determining how the results/outputs/answers from EFDSS should best be formulated and presented

- determining when and how the community would want to access the EFDSS

- determining who should be the local custodian of the EFDSS software.

\subsection{Visits to the Catchment}

A field trip to the Border Rivers Catchment was organised by the software developers to give them a feel for some of the issues facing community members who are actually impacted by management decisions. Numerous landholders and other interested community members were met and introduced to the project. This was a great way to establish contact and served to assure community members that the developers were genuine in their interest in the catchment and its issues. Although the focus of the project was not to develop a full application for the Border Rivers, it was imperative that the developers were familiar with the ecological condition and important ecological sites within the catchment. 


\subsection{Communication Sheets}

A series of 12 communication sheets were written during the project for the reference group. Some of these were purely background information, describing various components of the system and how they worked. Others targeted specific features of a component and requested feedback and comment about such things as their design, implementation and functionality. The timing of the communication sheets was rather random, though it was worked around the visits to the catchment.

\subsection{Newsletters}

To keep people informed and up to date with the system's development, three newsletters were circulated to a wide variety of interested people from a range of community and government organisations as well as numerous individuals. These newsletters proved critical in promoting EFDSS outside the Border Rivers.

\section{INCORPORATING CONTRIBUTIONS}

The design and structure of EFDSS was always intended to be very visual, guided by the principle that a picture paints a thousand words. However it was critical that the underlying theory and modelling principles be easily available and understood by users. This meant that there was a requirement for EFDSS not only to present a wide range of results but also to facilitate broad-based information delivery within the context of riverine ecology. Providing graphs and tabular reports seemed a useful way of addressing the first requirement and the provision of some kind of text retrieval database was seen as a solution to the second - this ultimately led to the development of EFDSSInfo. Another major area for reference group input was in the general look and feel of the package.

Because of the importance of knowledge transfer in the context of a DSS, a great deal of effort was put into the design of these components, ie presentation of results and explanation. The reference group was introduced to these components through the communication sheets and demonstrations.

\subsection{Presentation of Results}

As much of EFDSS developed in an iterative manner, many of the suggestions from the reference group were simply incorporated along the way. There was no format method implemented to evaluate them and add them to the system. Never the less after the first demonstration of the 
system, feedback led to some major design changes. These changes resulted in a more user-friendly interface. The Public User Interface has a fixed style of interface and leads the user through the various phases of exploration. There is little opportunity for the user to explore the results independently of what is provided. Thus the suite of graphs and charts provided had to be the 'best' ones for conveying the results to the non-technical user. The reference group found the initial proposals for the graphing to be exceedingly complex, abstract and difficult to interpret - their preference at this stage would have been for detailed text reports. Extensive discussion and further feedback resulted in a modification to the types of graphs presented and to the story which they told. Some of these modifications are shown. In hindsight, the main problem with initial screen was not their format, but their content. They were quite complex analyses of the results and were difficult to explain. The final graphs are much simpler analyses of the results, but may not provide the same depth of understanding as the first set of graphs. Overall, the main problems with the graphs were:

- it is difficult to make graphs intuitive;

- no matter how hard you try, people can't understand graphs without understanding the data;

- the use of categories (e.g. extreme, high, medium, low) works for a while, but ultimately it is subjective and must be explained.

\subsection{Inclusion of Explanation - EFDSSInfo}

Much of the focus of the EFDSS is on understanding the role of river flow regimes in shaping riverine ecosystems. This means that the user needs to have access to a wide variety of pertinent information. Although built into the standard EFDSS PUI interface, EFDSSInfo is a discrete stand-alone module solely dedicated to information delivery. It takes advantage of hypertext and other multimedia-based technologies, particularly for context sensitive links and for graphical representations, similar to those which enable documents to be published via the Internet on the World Wide Web.

EFDSSInfo can be thought of as a very large comprehensive electronic book. In it there are the equivalents of chapters and sections about many aspects of riverine ecosystems. There are text, photographs, diagrams, tables, charts etc. Theory is introduced, technical information explained and local catchments are described. Navigational aids are provided to assist the user find particular pieces of information in a sensible manner and to browse through the entire contents efficiently. As well as explaining ecological principles, EFDSSInfo provides two very important functions: 
- it contains a localised catchment-based description - ie it has real meaning to the local users of the system

- it relates ecological principles and the catchment-based description to the EFDSS - ie it promotes the role of the EFDSS.

The EFDSSInfo module proved to be more attractive to the reference group than the EFDSS! Members of the community involved in the decision-making process are keen to learn more about their catchment and ecological processes in general and EFDSSInfo was accepted as a wonderful tool for assisting them in this endeavour. While little community input is in the prototype, the module is designed to be updated by the local community.

\section{CONCLUSIONS}

This exercise in using community consultation in software design arose from the need to involve potential end users in the design of the system. The interface for software that is to be used in a public consultation and education role is critical and the developers sought assistance from a group of people involved in the water allocation and management planning exercise. The developers consider the final product to be a successful exercise in good interface design and the community reference group's feedback and reactions strongly influenced the changes in interface design that were made during the design process. Thus, the consultation process was considered successful from the viewpoint of the designers of the system. There are lessons to learn in the setting up of such a group. While formal guidelines cannot be drawn up, those initiating the consultation process need to acquire a balance between knowing what planning processes are going on in the catchment, and keeping their distance from them. In hindsight, the formation of the larger group, as recommended in the initial consultation paper, would have contributed more to the development of the EFDSS and ensured that it could be more firmly placed in the decision-making process. However, that was not possible for the case study.

The most important lesson is that the best way of getting feedback and input is via live demonstration, in an informal environment. Most visits to the catchment were made by one member of the development team who was not one of the programmers and consisted of two to three demonstrations with a small group of people. Though these visits required as much, if not more, effort than the writing and production of the communication sheets, they were much more rewarding, both personally and technically. Looking back, the communication sheets provide a wonderful history of the development of the project but they were a lot of work for little obvious gain. 
The inclusion of explanation is critical to the success of a DSS, but it is difficult to determine the right level of explanation for a prototype. The approach adopted in the EFDSSInfo module, whereby the system is standalone and users can easily add their own information, should be promoted. This approach allowed the developers to design and implement full functionality, though much of the knowledge base, particularly the local knowledge and descriptions, are not included.

A final evaluation of the success of this approach to software interface design cannot be made until the software is used in a decision-making environment. Though the reference group are comfortable with the interface, and indeed the contents of the EFDSS, they have not used it! This is a dilemma encountered when the software development sits outside the process.

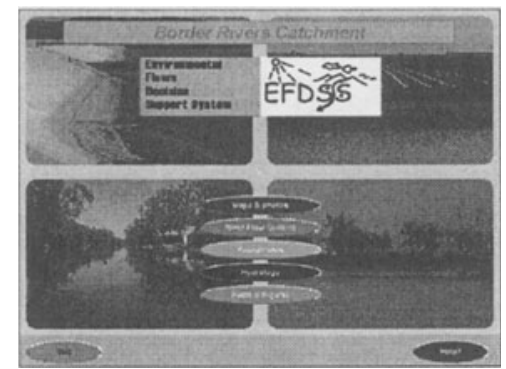

1. Original Assessment Screen

Reference group response indicated that this screen was somewhat abstract. It led to another 3 levels of action buttons to navigate to the actual assessment which was considered structurally too deep.

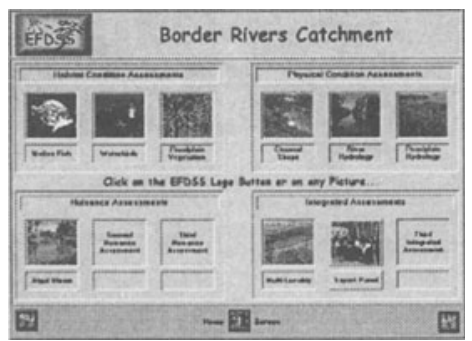

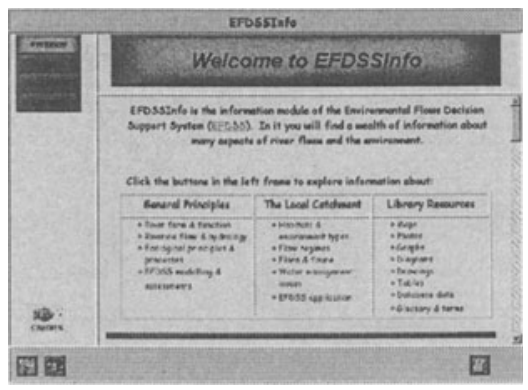

\section{EFDSSInfo}

The reference group liked this so much that they lobbied strongly for its inclusion in the EFDSS. They also wanted EFDSS to use similar styled buttons, header and footer panels and navigational aids. Many of these features were subsequently incorporated into EFDSS.

\section{Border Rivers Prototype \\ Many of the features requested by the reference group were incorporated into EFDSS particularly with a shallower navigational structure and layout.}

Figure 1

\section{REFERENCES}

Young, W.J., Booty, W.G., Whigham, P.A and Lam. D.C.L. (1999) Integrated assessments of river health using decision support software; this publication. 